\title{
The Janus Project: The Remaking of Nuclear Medicine and Radiology
}

\author{
Steven M. Larson
}

Nuclear Medicine Service, Memorial Sloan-Kettering Cancer Center, New York, New York

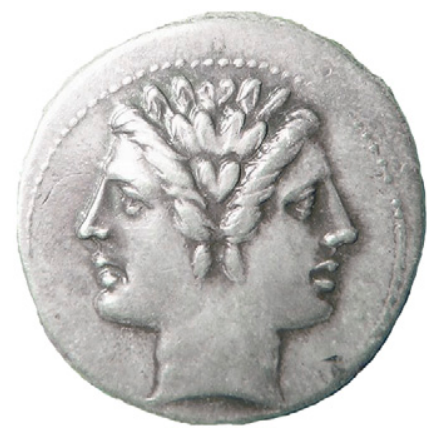

FIGURE 1. Janus coin.
In ancient Roman religion and mythology, Janus is the god of beginnings and transitions. He is depicted as having 2 faces on his head, facing opposite directions: one face looks eastward and the other westward, looking simultaneously into the future and the past. He also symbolizes change and transitions such as the progression of one condition to another, of one vision to another (Fig. 1) $(1,2)$.

I 1972, I took the first American Board of Nuclear Medicine (ABNM) examination, having satisfied requirements for training in nuclear medicine during fellowships and practical training in medical school and residency under my mentor, Wil B. Nelp, of the University of Washington, Seattle. I was attracted to the diversity of the field of nuclear medicine: radioimmunoassay had just been invented, opening a rich universe of understanding of internal homeostasis; ${ }^{131} \mathrm{I}$ and other therapies were the foundation of a strong endocrine and nuclear medicine component; cardiac physiology was being understood through radiotracer methods of perfusion and ventricular function; oncologic imaging was in its infancy, but tumor-specific processes such as antibody uptake by tumors promised to open a window on the pathophysiology of cancers. Major medical schools were adding expertise in nuclear medicine, and since this was a new field, the leaders were often youthful and dynamic individuals, who were stars in their parent disciplines, such as Henry Wagner at Johns Hopkins, Jim Adelstein at Harvard, and John McAfee at Syracuse.

When the ABNM was formed by agreement among a cadre of leaders in radiology, pathology, and nuclear medicine,

For correspondence or reprints contact: Steven M. Larson, Memorial Sloan-Kettering Cancer Center, 1275 York Ave., S-212, New York, NY 10021. E-mail: larsons@mskcc.org

COPYRIGHT (C) 2011 by the Society of Nuclear Medicine, Inc.

DOI: 10.2967/jnumed.110.085761 there was a great deal of both controversy and excitement over this promising new medical specialty. In those days, nuclear medicine physicians like me often compared ourselves to our colleagues in radiology, whom we saw as pretty much being interested in anatomy and higher salaries. We took pride in our broader medical orientation, knowledge of the tracer principle, and interest in physiology, function, and research. On the other hand, our radiologic colleagues were quick to note the deficiencies in the quality of our images"unclear medicine" was a common phrase-and they pointed with pride to the great impact made in medical practice by radiologic studies. In many ways, the strengths and personalities of the 2 specialties were already in opposition, and our practices were separate and distinct, especially in the first decade or so after formation of the ABNM.

In 2011, we have a totally transformed picture with regard to nuclear medicine. We find that a combination of the evolution of medical imaging practice and adverse economic factors has led to markedly diminished prospects for a strong and independent specialty of nuclear medicine, at least in the United States. In most major institutions in the United States, radiology exerts hegemony over imaging in determining both who will be hired into nuclear medicine practice and what the nuclear medicine practitioners will be credentialed to practice. Day-to-day nuclear medicine activities tend to resemble radiology practice, with less and less emphasis on direct patient care. The role of nuclear medicine in cardiology and thyroid therapy is diminishing. The ABNM's new standards for training, the 3-y curriculum, have not had the desired result, in part because radiology has not accepted them as being sufficient for cross-sectional credentialing.

Thus, nuclear medicine as a discipline, paradoxically at a time of huge scientific and clinical opportunities, is in serious trouble. There is great irony in the fact that a significant part of the problem comes from progress in medical imaging, namely the advances in quantitative molecular imaging and, in particular, the important role that cross-sectional imaging plays in modern instrumentation, such as PET/CT. With some justification, radiologists will say that nuclear medicine training is insufficient for the best understanding of modern cross-sectional imaging. On the other hand, the nuclear medicine community will respond that the private practice of nuclear medicine in 
the United States is largely in the hands of radiologists with an average of 4 mo of training in the specialty. Neither situation of training seems adequate for the demands of modern practice, and both specialties should go on record as supporting more complete training in these important areas.

\section{THE PROBLEM, PART 1: A CRISIS OF CONFIDENCE IN NUCLEAR MEDICINE TRAINING}

For nuclear medicine, the main problem is the bleak present for nuclear medicine-trained residents and the uncertain future for mid-career nuclear medicine-trained physicians without radiology backgrounds. I came to this realization when I read a letter sent from our nuclear medicine residents, Yang Lu, Ashima Lyall, Deepti Gupta, James Toby, and Aditi Shrutito, to Hedvig Hricak, chair of the Department of Radiology of MSKCC, in early 2011:

\begin{abstract}
"We, the Nuclear Medicine residents of the Department of Radiology at MSKCC wish to discuss with you our unique circumstance. We have exhausted both personal and local resources, and need your support in trying to address our common concerns regarding our professional futures. We have virtually no prospect of employment after completion of our training, which has included at the very least, medical school, internship, and a 3 year residency training program. We are training under a stressed horizon of financial loans and young families with growing needs. We desperately need your help to ensure that we have futures in clinical medicine once our training in Nuclear Medicine is complete.

"We are outlining the major problems which have obstructed our professional futures in Nuclear Medicine and offering a variety of possible solutions that we believe should be pursued in order to improve our complex situation. We have been fortunate to have a program here in Nuclear Medicine at MSKCC that is providing us with the best quality nuclear medicine training in the country, and have been busy, working diligently to improve our skills and CV's for our best chances for future successful professional futures. However, our situation is grim, and we come to you out of mutual helplessness and desperation.
\end{abstract}

"Our situation is not unique; it applies to nuclear medicine residents all over the United States. Aggressive steps are being taken to confront this time-sensitive matter, both by us and by individuals around the country. As members of your department, we need your support and for much more to be done or we face grave consequences. Our commitment to medicine and our training is unquestionable; we have all made large sacrifices to be where we are today.

"The problems:

"There are virtually no prospects of employment for current exclusively Nuclear Medicine board certified physicians. Available PET/CT and Nuclear Medicine positions are being awarded to board certified diagnostic radiologists with sometimes little to no formal PET/CT or Nuclear Medicine training.

"Despite the interconnectedness of the fields of Diagnostic Radiology and Nuclear Medicine, physicians certified in Nuclear Medicine alone are limited in what services they are allowed to bill or obtain certification for, despite equivalent training, greatly limiting our employability or relevance to our own careers in Nuclear Medicine.

"We are unfairly labeled in a variety of ways as unfavorable candidates when attempting to switch residencies or pursue additional training because we are Nuclear Medicine residents, despite the reputation for excellence we have acquired during our tenure here at MSKCC.

"Possible solutions:

"There must first be a realization of a potential great loss of talent and training in the Nuclear Medicine residents here at MSKCC, [who have] dedicatedly served a variety of services with incredible professionalism and talent over the last several years.
"Steps must be taken to convert the existing Nuclear Medicine residency program into a combined Nuclear Radiology program with dual board certification in Nuclear Medicine and Radiology. We must prevent more new residents from finding themselves in the same predicament while continuing an outstanding training program that provides necessary services to the hospital.

"Design an extended fellowship program here at MSKCC that is tailored to current Nuclear Medicine residents and bridges our training experiences and awards us with certification in diagnostic radiology.

"Combine current Nuclear Medicine residents as a group into another training program, as is being currently done between the Nuclear Medicine Residency program and the Radiation Oncology Program at the University of Pennsylvania.

"Evaluate Nuclear Medicine residents that have acquired equivalent training as compared with diagnostic radiology residents (in CT, for example) and create a certification process that assesses the competency of physicians in their abilities to interpret $\mathrm{CT}$, and provides them with recognized certification. Begin to take steps necessary on a national scale to fuse these 2 highly-integrated careers and encourage the combined involvement of Nuclear Medicine and Diagnostic Radiology researchers to uncover the vast potential of applying molecular imaging techniques and therapies into clinical practice.

"We hope we can speak together with you in person, and discuss these important issues."

Dr. Hricak and I were profoundly affected by this heartfelt plea that sets the problem in perspective as nothing else could. We both set out immediately to work, as we have on other difficult problems affecting our future, and were able to help our trainees get a slot for training in radiology or, in one case, a faculty appointment in nuclear medicine. But it was difficult and, frankly, extraordinary...the kind of thing that may work on a one-time basis only. In the long term, we realized that we needed a better plan that would resolve this dilemma permanently. Our trainees are our future, and we in nuclear medicine and indeed imaging sciences must do whatever we can to help them both as individuals and as a group. These individuals are well trained in an excellent program, one of the leading programs in the country, with a large clinical volume, modern equipment, and a strong research and academic environment. Other top-rank clinical/research nuclear medicine programs in my mind also include the Harvard program, Washington University (St. Louis), UCLA, Stanford, and Duke, but without exception, the same scenario is at play at these sites as in most nuclear medicine training programs sponsored by the Accreditation Council for Graduate Medical Education in the United States. Leadership in both radiology and nuclear medicine must do everything it can to avoid wasting the talent and energy of nuclear medicine trainees such as those at MSKCC. We must actively commit ourselves to seeking a solution to this problem.

\section{THE PROBLEM, PART 2: A WEAKNESS IN RADIOLOGY AS AN ACADEMIC DISCIPLINE}

I also believe that radiology, despite its economic strength and current hegemony over imaging, must move carefully if it is to have a rosy future as an academic discipline. For one thing, both the hegemony of radiology over imaging and the economic strength of the field are under threat. A recent article by Levin et al. reported a review of Medicare, Part B, payments over the last 2 decades. A significant portion of 
clinical imaging is not performed by radiologists but by other clinical specialists who seek advantage through control of patients to perform and interpret imaging of their own patients. Cardiologists are the next most common medical discipline to bill for noninvasive diagnostic imaging (NDI). The authors summarize their findings: "The growth in feefor-service payments to nonradiologists for NDI was considerably more rapid than the growth for radiologists between 1998 and 2006. Then, by the end of 2008, 2 years after the implementation of the Deficit Reduction Act, steeper revenue losses had been experienced by radiologists. The result was that by 2008, overall Medicare fee-for-service payments for NDI were $4 \%$ higher to nonradiologists than they were to radiologists" (3). In my opinion, the only way to counter this trend is to emphasize the improved quality of care that results when true experts interpret NDI studies, and this means correcting the serious deficiencies in our training programs.

Another big cloud on the horizon is that radiology is not producing enough young radiologists who are research leaders, because training has had such a sharply clinical focus, and also that there are too few radiologists who are outstanding research mentors. Of course, there are brilliant exceptions, such as Ralph Weissleder of Harvard, and Elias Zerhouni as a young faculty member at Hopkins, but these individuals are few and far between. With the growing clinical diversity of the field, time in training is becoming increasingly restricted to clinical disciplines, and imaging research has taken a back seat. Unlike the internal medicine and surgical subspecialties, there is not a strong tradition of research fellowships that are essentially a time-out from practical clinical training opportunities. With the growing demand to maintain the clinical workload so that salaries do not drop at a time of reimbursement reductions, this problem may grow worse. If this happens, radiology could slip into the status of a medical trade.

I mention a personal experience illustrating that the characteristic difference in research emphasis by radiology and internal medicine is longstanding. As a young faculty member with board certification in both nuclear medicine and internal medicine, I had occasion to visit the chairs of both departments. I recall a conversation, in the cafeteria of the University of Washington, with the chair of radiology, who offered the opinion that research should be done on evenings and weekends and that anyone who was really good would succeed with this research schedule. An alternative view was expressed by the chair of internal medicine at the Seattle Veterans Administration Hospital, where I was chief of nuclear medicine; the chair expected that at least $60 \%$ of one's time should be spent building a research program.

The problem is compounded by the fact that clinical reimbursement is going down while salary expectations continue to be high, meaning that imaging practitioners must spend more time reading scans as opposed to being engaged in scholarship or research. Of course, an inherent conflict is then created between the expectations that come from research aspirations on the one hand and money to pay for those noble sentiments on the other hand. I spent a lot more time in research over my career than most young faculty members in nuclear medicine or radiology will spend over theirs. An academic career is not for everyone, and we cannot expect to be paid from clinical dollars for research activities; taking everything together, in my career I certainly was paid less than someone in private practice. It follows, therefore, that on balance the salary of an imaging scientist academician will be less than that of someone devoted totally to clinical work. It is really time-time for research-that is the great value here.

I advise trainees who are serious about becoming physician-scientists that as their first priority, they should get into a faculty situation where there is significant time for research, and the trade-off will be salary. . .it has to be. If possible - and this is the happy situation for our young MD faculty in the human oncology pathogenesis program at Memorial SloanKettering Cancer Center (MSKCC) - they should spend 80\% of their time doing research and $20 \%$ doing clinical work. So commit to the excitement of research, but be prepared to give up the Escalade in the driveway.

There is no doubt that nuclear medicine has contributed directly and indirectly to advances in radiology. This fact has been widely recognized by radiology leadership. For example, James Thrall, MD, chair of radiology at Massachusetts General Hospital and board-certified by ABNM, has said, "I have always felt that nuclear medicine was the beacon for the rest of imaging. When I had the opportunity to give the Cassen lecture at the SNM, I realized that every advance in radiology was either done first in nuclear medicine or was put into practice first in nuclear medicine. As a department chair and 'nuc,' I feel as though I have had an unfair advantage because we already knew where things were going. After all, functional MRI is just a reprise of what we had done years before with PET, etc." (personal communication, 2011).

Also, leaders in the development of molecular imaging have been strongly influenced by nuclear medicine and the radiotracer techniques, and many of the new developments have come from a merger of tracer principles with other related imaging methods in a context of novel biology. For example, the In Vivo Center for Molecular Imaging initiative of the National Cancer Institute has incorporated this amalgam of approaches and has led to the enriched development of the new field of molecular imaging, with examples of tumor receptor imaging, bioluminescence imaging, quantitative immunoPET, gene expression imaging, photoacoustic imaging, and Raman spectroscopy.

At present, neither radiology nor nuclear medicine training is optimal preparation for a career of research excellence. Instead, a strong basic training in the clinical aspects, coupled with a dedicated fellowship experience in molecular imaging or targeted therapy, is needed to ensure optimal growth of the diagnostic imaging specialties over the long term. To the great credit of other medical disciplines such as internal medicine, medical oncology, and radiation oncology, this is precisely the model that they have chosen to follow. 
For both nuclear medicine and radiology, I believe that this is a time of great crisis on the one hand and great opportunity on the other. To me, there is much to be gained by making it a common cause to seize the exciting opportunities offered by molecular imaging. I think that much of radiology understands the value of PET, and we do not hear the phrase "unclear medicine" much anymore. There are also hopeful signs that many in nuclear medicine and in radiology agree on the importance of molecular imaging, to the extent of identifying the best of the molecular imagers as common heroes. Recently, Stanford University recognized a need to enrich the discipline of radiology through recruitment of physician-scientists by the appointment of Sanjiv Sam Gambhir as chair of radiology, whom Stanford announced as "internationally recognized for his incredible scientific contributions, and for training and educating the new generation of physicians and scientists focusing on molecular imaging." This move was made to strengthen a research tradition that had taken hold at the Stanford Department of Radiology. We all agree that Dr. Gambhir is an excellent example of a physician-scientist and has set a standard to which young research-oriented nuclear medicine physicians and radiologists can aspire. I think that everyone in the imaging community, regardless of orientation-radiology or nuclear medicine-can embrace the idea that the merger of biology and imaging into molecular imaging, and the prospects for molecular targeted therapy, call for our best efforts as physicians and scientists to take maximum advantage of the new horizons opening to us for improving patient care.

\section{A BRIGHTER FUTURE: RADIOLOGY AND NUCLEAR MEDICINE WORKING TOWARD A COMMON GOAL}

Nuclear medicine and indeed all imaging sciences, including radiology, are threatened by both internal and external forces that will diminish our role in health care and limit our ability to attract outstanding trainees and to optimize advances in imaging research. I propose that the solution is for nuclear medicine and radiology to combine their complementary skills to work toward a common goal of the multidisciplinary development of molecular imaging. The first step should be to optimize training in order to address the challenges of a molecular imaging future.

Medical imaging as such has begun to rapidly change in recent years with the growing recognition that treatment for many common tumor types - such as lung, colon, breast, and prostate-must be individualized to be successful. This pursuit of personalized medicine will likely shape the development of medical imaging. In this essay, we consider modern molecular imaging, its growing impact on the practice of oncology, and the benefits of a closer association with radiology, especially for training. The introduction of hybrid imaging for PET/CT has been universally successful as a true innovation and has dramatically altered practice for the better. As we think about future developments, we see that molecular imaging offers a multimodality future, in terms of both imaging instrumentation and probes. Using research advances as a starting point, we then create a thought map for how molecular imaging will contribute to individualized cancer care in the future. In this discussion, it should be clear why the reshaping of our specialties-both radiology and nuclear medicine-is inevitable.

\section{THE OPPORTUNITY}

\section{Hybrid Imaging}

"Hybrid imaging is defined as the fusion of two or more imaging technologies into a single, new form of imaging. Typically, this new form is synergistic - that is, more powerful than the sum of its parts" (4).

In 2009, a group of radiologists and nuclear medicine physicians assembled at the Radiological Society of North America in Chicago to discuss hybrid imaging and to review the current status, training, and privileging requirements for the future. The overwhelming consensus was that hybrid imaging is a critical component of future molecular imaging. PET/CT was the main focus of this effort, but other kinds of imaging, such as PET/MRI, clearly will also be important to include as we go forward with training and credentialing. These facts indicate that more training will be needed by both radiologists and nuclear medicine physicians but in different aspects of the technology. Throughout the world, dedicated PET instruments are rapidly being replaced by PET/CT units. Moreover, it is also clear that hybrid tracers will be an important component of our future endeavor.

For example, Figure 2 shows an image of a patient with castrate-resistant prostate cancer, as defined by rising prostate-specific antigen levels in the face of castrate levels of serum testosterone $(<30 \mathrm{ng} / \mathrm{mL})$. Whole-body imaging was performed with ${ }^{18} \mathrm{~F}-\mathrm{FDG}$ PET, and the next day, imaging was performed with ${ }^{18} \mathrm{~F}$-dihydrotestosterone, an analog of dihydrotestosterone, the most abundant androgen at the cellular level in patients with prostate cancer. The purpose of these studies was to compare castrate-resistant prostate cancer on a lesion-by-lesion basis, in order to examine the heterogeneity of radiotracer metabolism. Thus, we see how beautifully the companion CT scan allows for direct comparison of the 2 different tracers at distinct points in time. In particular, the ${ }^{18} \mathrm{~F}-\mathrm{FDG}$ image shows that there is no ${ }^{18} \mathrm{~F}-\mathrm{FDG}$ uptake at a site in the vertebrae where a sclerotic margin is observed around a central lucent region. On the ${ }^{18} \mathrm{~F}$-dihydrotestosterone scan, a hypermetabolic mass that takes up the androgen receptor binding tracer is evident at this site. The companion CT scan provides a high-resolution framework for the PET scan.

\section{Hybrid Molecular Tracers}

Numerous textbooks and articles have now extolled the virtues of tracers for molecular imaging. Through exquisite tracer chemistry, we can have a combination of relevant biology and imaging technology. Literally hundreds of radiotracers and perhaps equal numbers of optical tracers have been developed that target individual molecules of importance 


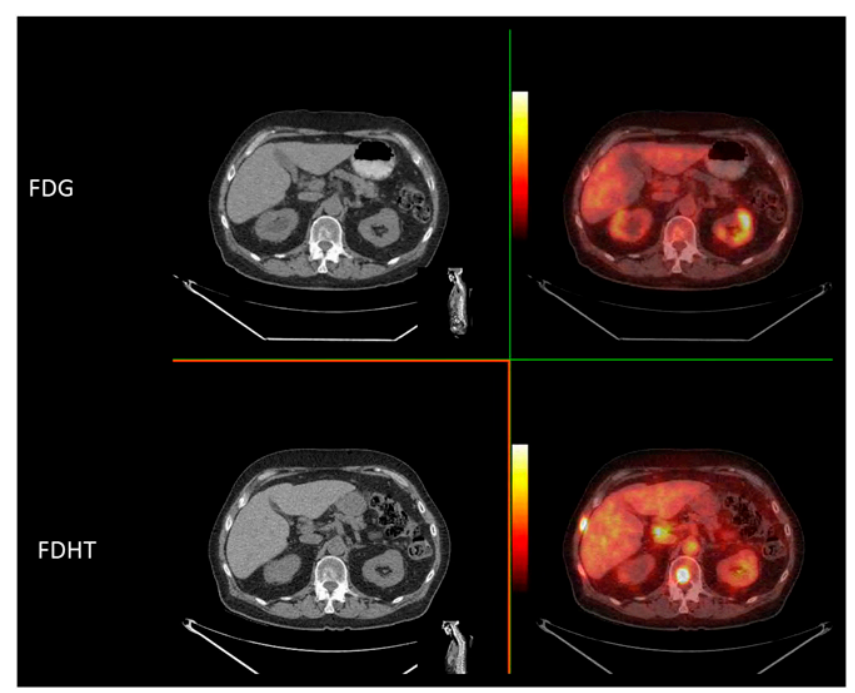

FIGURE 2. ${ }^{18} \mathrm{~F}$-FDG PET and ${ }^{18} \mathrm{~F}$-dihydrotestosterone PET images, along with companion CT scans, of patient with castrateresistant prostate cancer. Cross section through $\mathrm{T} 12$ reveals differential metabolism in body of vertebra. There is no uptake of ${ }^{18} \mathrm{~F}-\mathrm{FDG}$, but ${ }^{18} \mathrm{~F}$-dihydrotestosterone uptake is highly avid. FDHT = dihydrotestosterone.

to specific diseases. Categories of tracers include substrates for metabolism; anticancer drugs; tissue characteristics of malignancy, such as invasiveness, hypoxia, apoptosis, and angiogenesis; and antibodies against cancer-testes antigens, fetal antigens, and differentiation antigens. These tracers target specific molecules of pathophysiologic importance.

An important direction of development is optimization of hybrid imaging through the use of hybrid tracers, especially those for which a nanocarrier is the basis for the tracer. A case in point is the selective targeting by $\mathrm{C}$-dots covered with polyethylene glycol derivatives, recently reported by Benezra et al., which have both optical and PET detection capabilities (Figs. 3 and 4) (5).

There is huge versatility in the nanotech approach, and in principle this approach could be combined with MRI, Raman spectroscopy, fluorescence imaging, bioluminescence imaging, and PET if the right combination of nanoplatform and modifications is provided. Nanoplatforms have been used therapeutically for years to reduce the toxicity of anthracyclines and antifungal agents. Our understanding of the biology of targeting these interesting nanostructures will grow over the next few years, but I believe the possibilities will also include the combining of diagnostic and therapeutic features in the same platform.

\section{RESOLVING THE CRISIS OF CONFIDENCE IN NUCLEAR MEDICINE TRAINING}

\section{The Solution}

Recently, the American College of Radiology and American Board of Radiology (ABR) published guidelines for training requirements in nuclear medicine. The document is well written and contains much useful information about current thinking in radiology and future requirements for

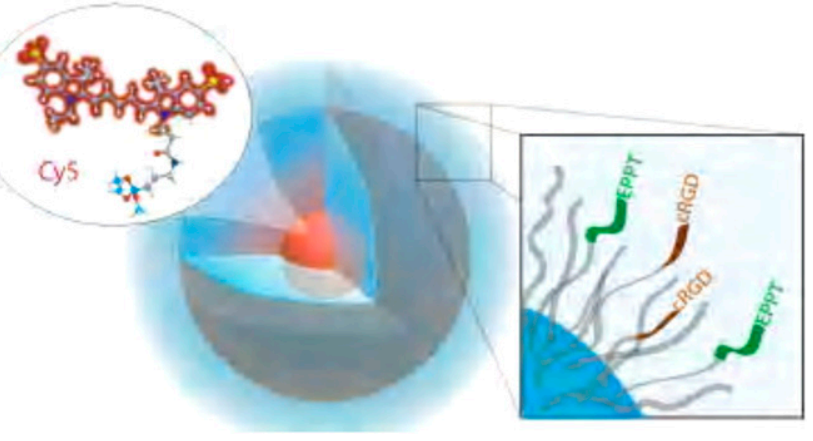

FIGURE 3. ${ }^{124}$ I-RGD-bound C dot. Highly constrained and intensely bright dye Cy5 is at core of silica coating that is $7 \mathrm{~mm}$ in diameter. In this case, tyrosine moieties are attached to polyethylene glycol on surface of particles and iodinated with ${ }^{124}$ I for PET. In this way, a true hybrid tracer is developed that has both optical and radionuclide detection properties.

training. The increase in flexibility offers a great opportunity with regard to training requirements within ABR-approved programs (6). It is this new flexibility that offers the single most important opportunity for combined training to meet the future needs of diagnostic imaging. Of course, the ABNM should take issue with the statement that radiologists are "uniquely" qualified for hybrid imaging. Current nuclear medicine training offers complete training to meet the needs of cross-sectional imaging in the hybrid setting, and training in the use of radioisotopes, including PET and therapy, is much more completely dealt with in the recommended nuclear medicine curriculum. Perhaps the most glaring deficiency is the lack of a recommendation to improve radiologist training in nuclear medicine and PET imaging before recommending

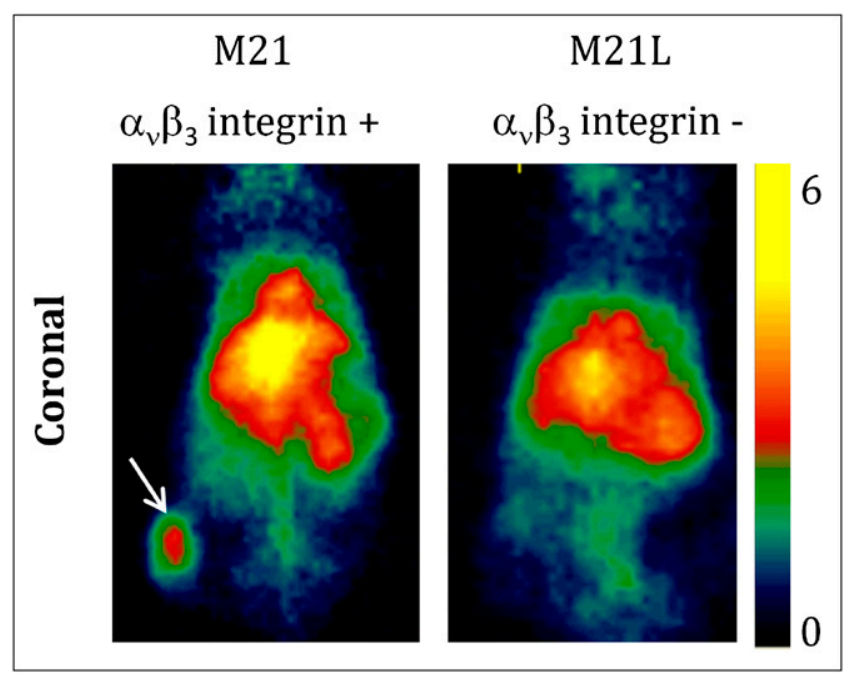

FIGURE 4. See Figure 3 for diagram of hybrid PET, optical silicabased nanoparticle. $\alpha_{v} \beta_{3}$ integrin is also attached to silica particles, and after injection into mice bearing melanomas expressing this integrin on their vasculature, there is uptake on M21 melanoma positive for integrin but no uptake within M21L melanoma negative for integrin. PET images are shown that document uptake into integrin-expressing melanoma, at arrow. 
practice. Quality will follow adequate training, and taking shortcuts would be unwise.

From the standpoint of nuclear medicine, the main deficiency in this document is that it does not offer a solution to the current problem that much of nuclear medicine practice depends on a cadre of radiologists whose training is more limited than described in the optimal nuclear medicine training pathways; namely, most practice is based on the minimum training described -4 mo-in nuclear medicine. This deficiency challenges the commitment of the American College of Radiology and ABR to the quality of practice at a time when well-trained nuclear medicine residents cannot obtain a job in clinical nuclear medicine practice, as described in the letter quoted earlier. These joint problems should be the subject of ABR and ABNM dialog.

Thus, we should start in earnest on the Janus project - the remaking of both nuclear medicine and radiology-to prepare ourselves for an amazing future of molecular imaging advances. Radiologists and nuclear medicine physicians should look on this time as one of transition and as the beginning of a renewal of our specialties. It is a time for change, a transition from one vision to another. A starting point can be a change in our way of thinking, to allow for better choices about the path forward. As an imaging community, can we make healthy changes to our approach and thus create some advantage from the unrest that is occurring?

\section{The Next Steps}

Our current status should allow for development of a combined pathway for new trainees that will lead to improved competence in advanced medical imaging and knowledge of molecular imaging. Adopting these proposed recommendations will go a long way toward resolving our current crises and preparing our disciplines for the future (Table 1).

We should begin with a new pathway for training: preparing physicians to be equipped for the new hybrid molecular imaging. The term nuclear medicine is really a synonym for molecular imaging and therapy. Clearly, our training must heavily emphasize the biology of the new targeted therapies so that we will have a better grasp of imaging opportunities for individualized medicine of the future. We can start with $\mathrm{ABR}$ requirements for $2.5 \mathrm{y}$ of basic training in the cross-sectional imaging modalities, CT and MRI, as a foundation in the anatomic training that will be the bridge between our disciplines.

A key point is that individuals emerging from a training program must be eligible for both the ABNM and the ABR and should have access to postresidency training fellowships across the board: in molecular imaging, MRI, mammography, interventional radiology, and, possibly, targeted therapies.

Finally, we recommend a fellowship in molecular imaging. A good fellowship is one that includes the excitement of discovery.

As a college student, I had worked as a radiochemistry technician in a laboratory that handled radioactivitycontaminated biologic samples from the atmospheric testing program in the Pacific, where tiny islands such as Enewetak were blown up and biologic consequences mapped for years afterward (7). I developed a fascination with radiotracers and sought out the chief of nuclear med-

TABLE 1

Combined ABNM-ABR Training Program in Molecular Imaging

\begin{tabular}{|c|c|}
\hline Recommendation & Description \\
\hline Radiology training & $\begin{array}{l}2.5 \text { y of training with emphasis on CT and MRI, including standard rotations in interventional radiology, } \\
\text { emergency radiology, and neurology (under auspices of ABR) }\end{array}$ \\
\hline $\begin{array}{l}\text { Nuclear medicine } \\
\text { training }\end{array}$ & 16 mo of training in basic nuclear medicine and $2 \mathrm{mo}$ in molecular imaging (under auspices of $\mathrm{ABNM}$ ) \\
\hline Additional training & $\begin{array}{l}\text { At end of } 4 \text { y of training, although eligible for } A B R \text { and } A B N M \text { certification, trainee is encouraged to take } 1 \text { - to } \\
2-y \text { fellowship training in molecular imaging }\end{array}$ \\
\hline \multirow[t]{6}{*}{$\begin{array}{l}\text { Molecular imaging } \\
\text { fellowships }{ }^{*}\end{array}$} & $\begin{array}{l}\text { Specialized molecular imaging core curriculum, weekly lectures on advanced imaging and probe } \\
\text { development, relevant biology in cancer and other target tissues }\end{array}$ \\
\hline & $\begin{array}{l}\text { Dual-mentor-supervised research program in molecular imaging } \\
\text { Basic science mentor from disciplines of biochemistry, pharmacology, immunology, cell biology, } \\
\text { molecular biology, genetics }\end{array}$ \\
\hline & $\begin{array}{l}\text { Molecular imaging mentor with emphasis on radiochemistry, cancer pharmacology, tumor immunology, } \\
\text { gene therapy, imaging physics }\end{array}$ \\
\hline & $\begin{array}{l}\text { Practica in technical aspects of molecular imaging such as small-animal imaging, vector development, and } \\
\text { radiolabeling }\end{array}$ \\
\hline & $\begin{array}{l}\text { Access to core curriculum of parent university MD-PhD program in cell biology, pharmacology, animal } \\
\text { handling, research ethics, statistics }\end{array}$ \\
\hline & $\begin{array}{l}\text { Weekly institutional lecture series, including molecular imaging series with outside experts, cancer biology, } \\
\text { and translational cancer biology }\end{array}$ \\
\hline
\end{tabular}

*Modified from R25T Molecular Imaging Fellowship at MSKCC. 
icine at the University of Washington, Wil B. Nelp, to discuss specialized training. I worked summers and took a 1-y fellowship in nuclear medicine while I was in medical school. It was an early version of molecular imaging, without PET. I put my radiochemistry background to use, and I developed what I think is the first kit introduced into nuclear medicine, and one of the earliest nanoformulations, a ${ }^{99 \mathrm{~m}} \mathrm{Tc}$-sulfer colloid kit that is still in use today, $45 \mathrm{y}$ later, for lymphoscintigraphy and reticuloendothelial system imaging. Here is how we described it in the original article: "For routine preparation of the colloid in our laboratory, a colloid kit is assembled consisting of three units, a $30 \mathrm{cc}$. multidose vial containing a four $\mathrm{ml}$. 'pre-mix' aliquot of rhenium, thiosulfate and Dextran; a sterile disposable syringe with one $\mathrm{ml}$. of $1 \mathrm{~N} \mathrm{HCI}$, and a disposable syringe with three $\mathrm{ml}$. of sterile phosphate buffer mixture. A calibrated aliquot of ${ }^{99 \mathrm{~m}} \mathrm{Tc}$-saline eluent is obtained from the ${ }^{99}$ Mo generator and added to the 'pre-mix', acidified, and heated for three minutes, after which it is neutralized in the phosphate buffer and cooled. Because the colloid is stable for at least 24 hours, it can be prepared each morning with adequate ${ }^{99 m}$ Tc activity to last throughout the day" (8).

The time is ripe for a formal fellowship in molecular imaging for incoming trainees with combined ABNM/ABR eligibility. This program should have both clinical and basic aspects, with a strong emphasis on "discovery" research. Clinical applications of molecular imaging are growing, with ${ }^{18}$ F-FDG PET/CT being the model for other tracers and other fusion imaging approaches. The NCI-sponsored P50 grants, In Vivo Cellular and Molecular Imaging Center (ICMIC), have now been awarded to a total of 7 centers. Several of these, including the original 3, have been in operation for more than 10 years as P50 SPORE type programs (Specialized Programs of Research Excellence). These programs have energized molecular imaging. ICMICs have led to numerous new tracers and have helped establish a culture of investigative excellence in molecular imaging. Also, several centers have established training grants, such as the MSKCC R25T, that can serve as a model for a molecular imaging fellowship. For example, under the leadership of Dr. Hedvig Hricak, the radiology/nuclear medicine research faculty at MSKCC has put together a program that has now trained 18 imaging scientists, 14 of whom are MDs, PhDs, or DOs. These trainees are already having an impact on new grant development and translation of molecular imaging from laboratory to clinic.

\section{CONCLUSION}

The approach that I have proposed in this essay is for nuclear medicine and radiology, in a true merger of purpose, to create a common cause around the topic of molecular imaging and therapy. I suggest that Janus is a good symbol of this merger, for several reasons: both groups will never be quite the same but will have enhanced capabilities, assimilating the best, even though contradictory, characteristics of each discipline into a new entity with enhanced capability for molecular imaging and improved clinical and research implications. A new training pathway will emerge that will combine the strengths of the 2 disciplines: the physiologic orientation of nuclear medicine and the anatomic orientation of radiology. In this way, we will take advantage of the lessons of the past while looking with informed sight toward a brighter future.

\section{ACKNOWLEDGMENTS}

I am grateful for the input from nuclear medicine trainees and colleagues that has led to this essay and related recommendations. Specifically, conversations with Dr. Hedvig Hricak, James Thrall, John Humm, Jason Lewis, Jorge Carrasquillo, Henry Royal, David Mankoff, Michelle Bradbury, Bill Strauss, and Joe Osborne were helpful in formulating this opinion piece. No potential conflict of interest relevant to this article was reported.

\section{REFERENCES}

1. Janus. Wikipedia: The Free Encyclopedia. Available at: http://en.wikipedia.org/ wiki/Janus. Accessed November 4, 2011.

2. Wittner P. Facing Two Ways. GenericsWeb. Available at: http://www.genericsweb. com/index.php?object_id=1011. Accessed November 15, 2011.

3. Levin DC, Rao VM, Parker L, Frangos AJ, Sunshine JH. Medicare payments for noninvasive diagnostic imaging are now higher to nonradiologist physicians than to radiologists. J Am Coll Radiol. 2011;8:26-32.

4. Hricak H, Choi BI, Scott AM, et al. Global trends in hybrid imaging. Radiology. 2010;257:498-506.

5. Benezra M, Penate-Medina O, Zanzonico PB, et al. Multimodal silica nanoparticles are effective cancer-targeted probes in a model of human melanoma. J Clin Invest. 2011;121:2768-2780.

6. ACR/ABR clinical statement on credentialing and privileging of radiologists for diagnostic nuclear medicine, including multimodality hybrid imaging. JACR. 2011;8:617-621.

7. Enewetak Atoll. Wikipedia: The Free Encyclopedia. Available at: http://en. wikipedia.org/wiki/Enewetak_Atoll. Accessed November 8, 2011.

8. Larson SM, Nelp WB. Radiopharmacology of a simplifield technetium-99mcolloid preparation for photoscanning. J Nucl Med. 1966;7:817-826. 